09

\title{
Предельно короткие оптические импульсы в углеродных нанотрубках и поглощение на тяжелых ионах
}

\author{
(C) Н.Н. Конобеева ${ }^{1}$, М.Б. Белоненко ${ }^{1,2}$ \\ ${ }^{1}$ Волгоградский государственный университет, \\ 400062 Волгоград, Россия \\ ${ }^{2}$ Волгоградский институт бизнеса, \\ 400048 Волгоград, Россия \\ e-mail: yana_nn@volsu.ru
}

Поступила в редакцию 07.02.2018 г.

В окончательной редакции 09.04.2018 г.

\begin{abstract}
Исследовано распространение электромагнитных волн в среде с зигзагообразными углеродными нанотрубками. На основании уравнений Максвелла получено эффективное уравнение для вектор-потенциала электромагнитного поля, в котором учтены колебания ионов диэлектрической среды, в которую помещены нанотрубки. Выявлена зависимость формы импульса от характера колебаний тяжелых ионов, на которых может происходить поглощение электромагнитного поля, а также от других параметров задачи.
\end{abstract}

DOI: $10.21883 /$ OS.2018.09.46557.41-18

Взаимодействие электромагнитного излучения с веществомактивно изучается и находит множество практических приложений [1-5]. Особенное внимание исследователей привлекают предельно короткие оптические импульсы, обладающие такими примечательными свойствами, как высокая направленность и высокая интенсивность излучения $\left(10^{21} \mathrm{~W} / \mathrm{cm}^{2}\right)[6]$.

Развитие инжекционных полупроводниковых лазеров накачки, элементов волоконной оптики и эффективных преобразователей лазерного излучения во вторую гармонику позволило создать исключительно компактные системы. В качестве примера можно привести лазерную установку, излучающую импульсы длительностью $180 \mathrm{fs}$, при этом средняя мощность составляет $10 \mathrm{MW}$ при частоте следования $50 \mathrm{MHz}$. При этом размеры прибора сравнимы по величине с ладонью [6]. Отметим также, что прогресс в данном направлении обеспечивает и использование наноструктур, которые активно применяются при создании таких устройств.

В настоящей работе речь пойдет об углеродных нанотрубках (УНТ), нелинейные свойства которых давно интенсивно изучаются [7-9]. В том числе интенсивно исследуется и динамика предельно коротких оптических импульсов или световых пуль в данных средах [10-13]. В частности, были получены эффективные уравнения, изучена динамика импульсов с учетом влияния примесей и кулоновского взаимодействия электронов, а также процессы столкновения световых пуль и влияние внешних полей.

Остался нерассмотренным вопрос влияния колебаний тяжелых ионов среды, в которую помещены углеродные нанотрубки, и поглощения ими электромагнитного поля. Учет данного воздействия важен при исследовании распространения предельно коротких оптических импульсов в нелинейных дисперсионных средах, что подтверждается, например, работами [14,15]. Поглощение на таких ионах может значительно сузить диапазон прозрачности и уменьшить ширину спектра пропускания в среде с углеродными нанотрубками.

\section{Постановка задачи и основные уравнения}

Рассмотрим распространение предельно коротких электромагнитных импульсов в углеродных нанотрубках. Электрическое поле импульса направим вдоль оси нанотрубок.

Закон дисперсии УНТ типа зиг-заг

$$
\varepsilon_{s}(p)= \pm \gamma \sqrt{1+4 \cos (a p) \cos (\pi s / m)+4 \cos ^{2}(\pi s / m)}
$$

$s=1,2, \ldots, m$, нанотрубка имеет тип $(m, 0), \gamma \approx 2.7 \mathrm{eV}$, $a=3 b / 2 \hbar, b=0.142 \mathrm{~nm}$ - расстояние между соседними атомами углерода.

Как следует из правил квантовой механики в присутствии внешнего электрического поля $\mathbf{E}$, которое будет рассматриваться в калибровке: $\mathbf{E}=-\frac{\partial \mathbf{A}}{c \partial t}$, необходимо заменить импульс на обобщенный импульс: $q \rightarrow q-e A / c$ ( $e$ - заряд электрона, $c$ - скорость света).

Уравнения Максвелла с учетом калибровки можно записать в следующем виде [16]:

$$
\frac{\partial^{2} \mathbf{A}}{\partial x^{2}}+\frac{\partial^{2} \mathbf{A}}{\partial y^{2}}+\frac{\partial^{2} \mathbf{A}}{\partial z^{2}}-\frac{1}{c^{2}} \frac{\partial^{2} \mathbf{A}}{\partial t^{2}}+\frac{4 \pi}{c} \mathbf{j}=\frac{4 \pi}{c} \frac{\partial \mathbf{P}}{\partial t} .
$$

Вектор-потенциал считается имеющим вид $\mathbf{A}=(0,0, A(x, y, z, t)), \quad$ a $\quad$ ток $\quad \mathbf{j}=(0,0, j(x, y, z, t))$. Для учета свойств среды мы дополнили уравнение (2) слагаемым с поляризацией среды $\mathbf{P}$, направленной вдоль 


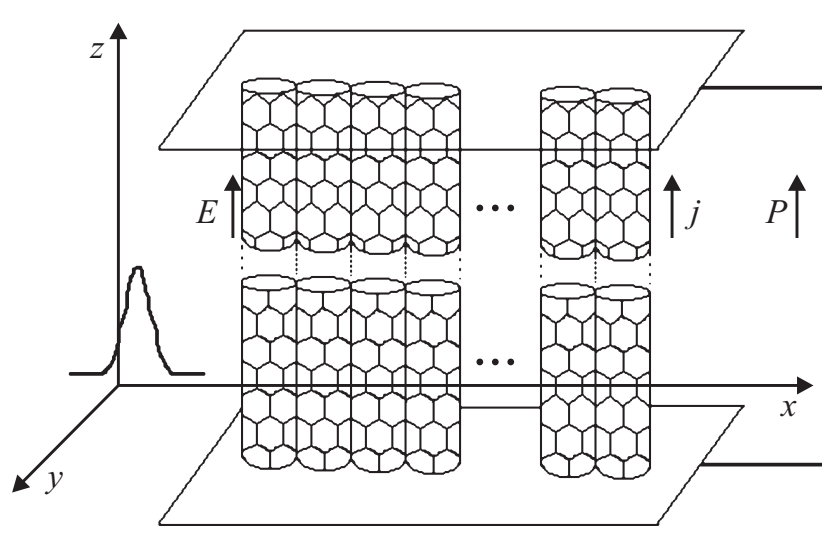

Рис. 1. Геометрия задачи.

оси УНТ (рис. 1). Запишем стандартное выражение для плотности тока

$$
j=e \sum_{p s \sigma} v_{s}\left(p-\frac{e}{c} A(x, y, z, t)\right)\left\langle C_{p s \omega}^{+} C_{p s \sigma}\right\rangle,
$$

где $v_{s}(p)=\frac{\partial \varepsilon_{s}(p)}{\partial p}$, а скобки означают усреднение с неравновесной матрицей плотности $\rho(t) .\langle B\rangle=$ $=S p(B(0) \rho(t)) . \quad C_{p s}^{+}, C_{p s}-$ операторы рождения, уничтожения возбуждений с квазиимпульсом $(p, s)$. Учитывая, что $\left[C_{p s \sigma}^{+} C_{p s \sigma}, H\right]=0$, из уравнений движения для матрицы плотности сразу получаем, что $\left\langle C_{p s \sigma}^{+} C_{p s \sigma}\right\rangle=\left\langle C_{p s \sigma}^{+} C_{p s \sigma}\right\rangle_{0}$, где $\langle B\rangle_{0}=S p(B(0) \rho(0))$, $\rho_{0}=\exp (-H / k T) / S p(\exp (-H / k T)), \quad k-$ постоянная Больцмана, $T$ - температура. Закон дисперсии $\varepsilon_{s}(p)$ можно представить в виде ряда Фурье

$$
\begin{gathered}
\varepsilon_{s}(p)=\frac{1}{2 \pi} \sum_{s q} a_{s q} \cos (a p q), \\
a_{s q}=\int d p \cdot \cos (a p q) \varepsilon_{s}(p),
\end{gathered}
$$

где интегрирование проводится по первой зоне Бриллюэна, а $q$ - натуральное число. В этом случае из (3), (4) следует одно эффективное уравнение на вектор-потенциал $\mathbf{A}$, которое в цилиндрической форме может быть записано как

$$
\begin{aligned}
\frac{1}{r} & \frac{\partial A}{\partial r}\left(r \frac{\partial A}{\partial r}\right)+\frac{\partial^{2} A}{\partial z^{2}}-\frac{1}{c^{2}} \frac{\partial^{2} A}{\partial t^{2}} \\
& +\frac{4 e n_{0}}{c} \sum_{q=1}^{\infty} q b_{q} \sin \left(\frac{a e(A+\eta)}{c}\right)-\beta \frac{\partial P}{\partial t}=0 \\
b_{q}= & \sum_{s} a_{s q} \int d p \cdot \cos (a p q) \frac{\exp \left(-\varepsilon_{s}(p) / k T\right)}{1+\exp \left(-\varepsilon_{s}(p) / k T\right)}
\end{aligned}
$$

и интегрирование ведется в пределах первой зоны Бриллюэна. Здесь производная по углу в силу цилиндрической симметрии равна нулю. Неоднородность поля вдоль некоторой оси приводит к неоднородности тока, вследствие чего происходит накопление заряда в какой-то области. Проведенные расчеты показали, что эффектом накопления заряда для фемтосекундных импульсов можно пренебречь [13]. Отметим, что вследствие убывания коэффициентов $b_{q}$ с ростом $q$ в сумме в уравнении (5) можно ограничиться первыми 15 неисчезающими слагаемыми [17], и получить широко применяемый в приложениях, но не интегрируемый методом обратной задачи рассеяния аналог уравнения sine-Gordon [18].

Величина $\eta$ в (5) связана с ненулевой компонентой вектора смещений среды $u$ как

$$
\eta=-c d \int_{-\infty}^{t} \frac{\partial u\left(z, t^{\prime}\right)}{\partial z} d t^{\prime}
$$

Здесь мы рассматриваем самую простую модель, когда в среде возникает поляризация, линейная по приложенному электрическому полю и направленная параллельно этому электрическому полю вследствие пьезоэффекта

$$
P=d \frac{\partial u}{\partial z}
$$

$d$ - здесь и выше соответствующий пьезомодуль.

Уравнение (5) в этом случае необходимо дополнить уравнением на компоненту вектора смещений $u[19,20]$

$$
\frac{\partial^{2} u}{\partial t^{2}}+\gamma \frac{\partial u}{\partial t}+\omega_{0}^{2} u=\chi \frac{\partial A}{\partial t} .
$$

Здесь $\gamma$ - коэффициент поглощения на тяжелых ионах, $\omega_{0}-$ резонансная частота колебаний тяжелых ионов, $\chi$ - коэффициент восприимчивости.

В рамках описанной модели необходимо сделать ряд замечаний. Так, мы учитываем только одну компоненту вектора смещений, что очевидно может быть легко обобщено. Также мы не учитываем, что среда может обладать нелинейными акустическими свойствами и вектор поляризации может быть в итоге неколлинеарен вектору электрического поля.

\section{Результаты численного моделирования}

Исследуемые уравнения (5), (8) были решены численно с помощью прямой разностной схемы типа крест [21]. Начальное условие выбиралось в виде предельно короткого импульса, состоящего из двух полуколебаний электрического поля и может быть записано в следующем виде:

$$
\begin{gathered}
A(z, r, 0)=Q \cdot \exp \left(-\frac{\left(z-z_{0}\right)^{2}}{\gamma_{z}}\right) \exp \left(-\frac{r^{2}}{\gamma_{r}}\right) \\
\frac{d A(z, r, 0)}{d t}=2 Q u \frac{\left(z-z_{0}\right)}{\gamma_{z}} \exp \left(-\frac{\left(z-z_{0}\right)^{2}}{\gamma_{z}}\right) \exp \left(-\frac{r^{2}}{\gamma_{r}}\right) \\
u(z, r, 0)=0, \quad \frac{d u(z, r, 0)}{d t}=0
\end{gathered}
$$

где $r$ - радиус, $Q$ - амплитуда, $\gamma_{z}, \gamma_{r}$ определяют ширину импульса, $z_{0}-$ начальное смещение центра импульса. 


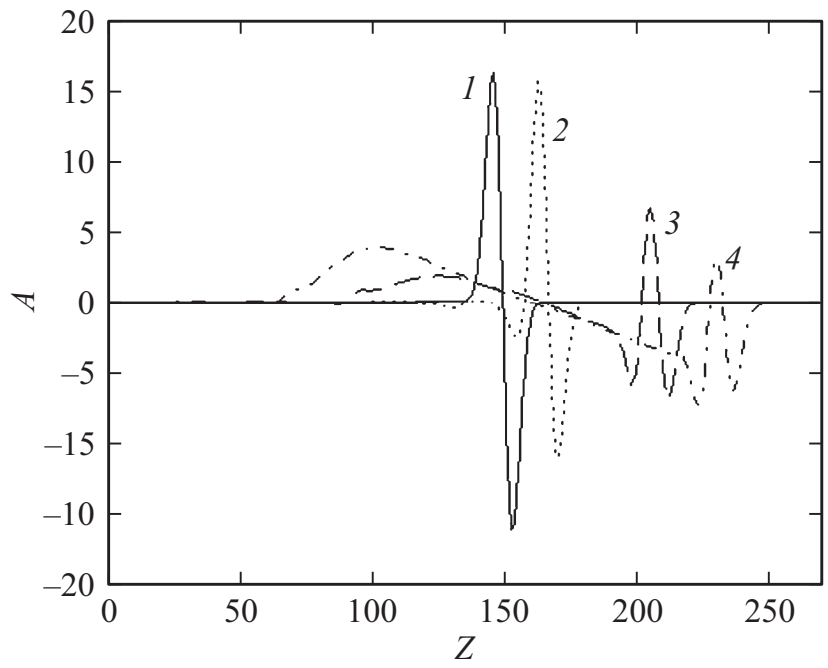

Рис. 2. Зависимость вектор-потенциала от координаты при $r=0$ : исходная форма импульса $(1) ; t=2 \cdot 10^{-13} \mathrm{~s}$ (2); $t=7 \cdot 10^{-13} \mathrm{~s}(3) ; t=10^{-12} \mathrm{~s}(4) ; v / c=0.95$.

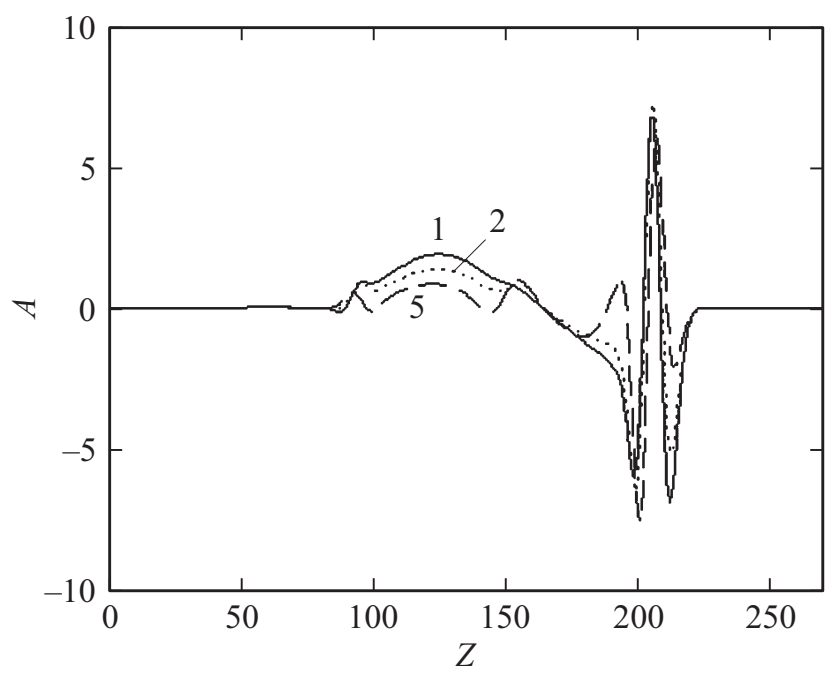

Рис. 3. Зависимость формы импульса от времени для величин константы связи, $d=1,2,5$ при $r=0$.

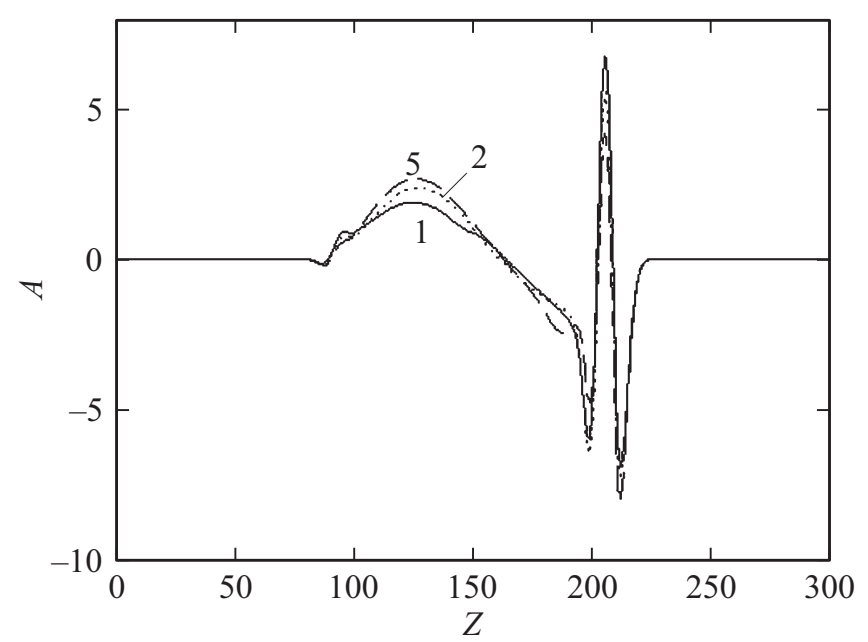

Рис. 4. Зависимость вектор-потенциала поля от времени для значений коэффициента поглощения на тяжелых ионах $\gamma=1,2,5$.
Возникающая эволюция электромагнитного поля при его распространении по образцу представлена на рис. 2.

Ввиду того, что уравнение (5) достаточно близко к интегрируемому уравнению sin-Gordon, импульс распространяется, сохраняя свою форму на первоначальном этапе, а далее начинают играть роль неинтегрируемые слагаемые в (5) и за импульсом появляется хвост, который имеет приблизительно нулевую площадь. Такое поведение, на наш взгляд, связано с тем, что в системе приближенно выполняется теорема площадей и потому возмущения, вносимые неинтегрируемой частью уравнения (5), не изменяют суммарную площадь под импульсом.

Стоит отметить также, что существует затухание амплитуды импульса при его распространении по образцу.

Зависимость формы импульса от константы связи $d$ представлена на рис. 3 .

Как следует из рис. 3, данный параметр влияет в основном на форму „хвоста“, следующего за импульсом. Но в отличие от случая без учета движения ионов, здесь наблюдается и изменение формы основного импульса. Причем чем больше величина $d$, тем сильнее колебания в „хвосте импульса“, вследствие пьезоэффекта и тем больше амплитуда основного импульса. То есть можно управлять режимом генерации терагерцовых импульсом, подбирая среду с тем или иным пьезомодулем $d$.

Влияние коэффициента поглощения на тяжелых ионах на процесс распространения импульса по образцу показано на рис. 4.

Из рисунка видно, что увеличение данного параметра приводит к заметному уменьшению амплитуды основного импульса и одновременному возрастанию площади под кривой „хвоста“.

\section{Заключение}

1. С течением времени наблюдается затухание амплитуды предельно короткого оптического импульса в пьезоэлектрической среде с УНТ, чего не наблюдалось ранее (без учета колебания ионов среды).

2. Управление „хвостом“ за предельно коротким импульсом осуществляется с помощью параметров $d$ и $\gamma$, что весьма важно при генерации терагерцовых импульсов.

3. Таким образом, учет колебания тяжелых ионов среды оказывает существенное влияние на характер распространения электромагнитных импульсов в массиве УНТ.

Работа поддержана Российским фондом фундаментальных исследований (проект № 16-07-01265-А). Численное моделирование с использованием параллельных технологий выполнено при финансовой поддержке Минобрнауки РФ в рамках государственного задания (проект № 2.852.2017/4.6).

Авторы выражают глубокую признательность С.А. Козлову за полезные дискуссии, которые стимулировали написание настоящей работы. 


\section{Список литературы}

[1] Schiffrin A., Paasch-Colberg T., Karpowicz N., Apalkov V., Gerster D., Muhlbrandt S., Korbman M., Reichert J., Schultze M., Holzner S., Barth J.B., Kienberger R., Ernstorfer R., Yakovlev V.S., Stockman M.I., Krausz F. // Nature. 2013. V. 493. P. 70. doi 10.1038/nature 11567

[2] Schultze M., Bothschafter E.M., Sommer A., Holzner S., Schweinberger W., Fiess M., Hofstetter M., Kienberger R., Apalkov V., Yakovlev V.S., Stockman M.I., Krausz F. // Nature. 2013. V. 493. P. 75. doi $10.1038 /$ nature 11720

[3] Apalkov V., Stockman M.I. // Phys. Rev. B. 2012. V. 86. P. 165118. doi 10.1103/PhysRevB.86.165118

[4] Kelardeh H.K., Apalkov V., Stockman M.I. // Phys. Rev. B. 2015. V. 91. P. 045439. doi 10.1103/PhysRevB.91.045439

[5] Goorjian P.M. Photonic switching devices using light bullets. 1997. Patent number: 5,651,079.

[6] Козлов С.А., Самариев В.В. Основы фемтосекундной оптики. М.: Физматлит, 2009. 292 с.

[7] Dresselhaus M.S., Dresselhaus G., Eklund P.C. Science of fullerenes and carbon nanotubes. San Diego: Academic Press, 1996. $965 \mathrm{p}$.

[8] Лозовик Ю.Е., Попов А.М. // УФН. 1997. Т. 165. С. 751. doi 10.3367/UFNr.0167.199707d.0751

[9] Елецкий А.В. // УФН. 2000. Т. 170. С. 113. doi 10.3367/UFNr.0170.200002a.0113

[10] Leblond H., Mihalache D. // Phys. Rev. A. 2012. V. 86. P. 043832. doi 10.1103/PhysRevA.86.043832

[11] Yanyushkina N.N., Belonenko M.B., Lebedev N.G., Zhukov A.V., Paliy M. // Int. J. Mod. Phys. B. 2011. V. 25. P. 3401. doi 10.1142/S0217979211101818

[12] Belonenko M.B., Popov A.S., Lebedev N.G., Pak A.V., Zhukov A.V. // Phys. Lett. A. 2011. V. 375. P. 946. doi 10.1016/j.physleta.2010.12.071

[13] Zhukov A.V., Bouffanais R., Fedorov E.G., Belonenko M.B. // J. Appl. Phys. 2013. V. 114. P. 143106. doi 10.17586/22208054-2016-7-6-936-940

[14] Kapoyko Y.A., Drozdov A.A., Kozlov S.A., Zhang X. // Phys. Rev. A. 2016. V. 94. N 3. P. 033803. doi 10.1103/PhysRevA.94.033803

[15] Kapoiko Y.A., Kozlov S.A. // Opt. Spectrosc. 2015. V. 119. N 3. P. 485-496. doi 10.1134/S0030400X15090131

[16] Ландау Л.Д., Лифиии, Е.М. Теория поля. М.: Физматлит, 1988. 512 c

[17] Белоненко М.Б., Глазов С.Ю., Лебедев Н.Г., Мещерякова H.E. // ФТТ. 2009. Т. 51. В. 8. С. 1657.

[18] Солитоны / Под ред. Буллаф Р., Кодри Ф. М.: Мир, 1983. $408 \mathrm{c}$.

[19] Ландау Л.Д., Лифиии, Е.M. Теория упругости. М.: Наука, 1987. $248 \mathrm{c}$.

[20] Блини, Р., Жеки Б. Сегнетоэлектрики и антисегнетоэлектрики. М.: Мир, 1975. 398 с.

[21] Бахвалов Н.С. Численные методы (анализ, алгебра, обыкновенные дифференциальные уравнения). М.: Наука, 1975. $632 \mathrm{c}$. 